\title{
Vena cava superior izquierda persistente. Implicaciones en la cateterización venosa central
}

\author{
Persistent left superior vena cava. \\ Implications in central venous catheterisation
}

\author{
G. Lacuey, M. Ureña, J. Martínez Basterra, N. Basterra
}

\section{RESUMEN}

La colocación de catéteres centrales por vía venosa subclavia y yugular se puede complicar con la canalización de una arteria o de una vía venosa aberrante. La anomalía más frecuente del desarrollo embriológico de la vena cava es la persistencia de la vena cava superior izquierda (VCSI). La implantación de catéteres en la VCSI se puede sospechar por el recorrido anómalo del mismo en la radiografía de tórax. La gasometría y la curva de presión del vaso permiten descartar una cateterización arterial. La confirmación diagnóstica se obtiene mediante angiografía, ecocardiografía, tomografía computerizada o cardio-resonancia.

El médico que implanta habitualmente catéteres venosos centrales, debe estar familiarizado con la anatomía del sistema venoso, sus variantes y sus anomalías, ya que su presencia puede influir en el manejo del paciente.

Palabras clave. Persistencia de vena cava superior izquierda. Retorno venoso sistémico anómalo. Catéter venoso central. Catéter Swan-Ganz.

\begin{abstract}
The placement of central catheters through the subclavian and jugular venous path can be complicated by the cannulation of an artery or an aberrant venous path. The most frequent anomaly of the embryological development of the caval vein is the persistence of the left superior vena cava (LSVC). The implantation of catheters in the LSVC can be suspected by its anomalous route in thorax radiography. Gasometry and the pressure curve of the vessel make it possible to rule out an arterial catheterisation. Diagnostic confirmation is obtained through angiography, echocardiography, computerised tomography or cardiac resonance.

The doctor who regularly implants central venous catheters must be familiar with the anatomy of the venous system and its variants and anomalies, since their presence might influence the handling of the patient.
\end{abstract}

Key words. Persistence of left superior vena cava. Anomalous systemic venous return. Central venous catheter. Swan-Ganz catheter.
Unidad Coronaria. Área del Corazón. Servicio de Cardiología. Hospital de Navarra. Pamplona.

Recepción el 7 de noviembre de 2008 Aceptación provisional el 19 de enero de 2009 Aceptación definitiva el 20 de enero de 2009

\section{Correspondencia:}

Gemma Lacuey Lecumberri

Mayor, $81, \mathrm{P}^{1}{ }^{\mathrm{O}}$

31310 Carcastillo (Navarra)

Tfno. 676713558

E-mail: g_lacuey@yahoo.es 


\section{INTRODUCCIÓN}

La cateterización de vías venosas centrales constituye una práctica habitual en las Unidades de Cuidados Coronarios, Cuidados Intensivos, Anestesia y Hemodiálisis. El retorno venoso sistémico presenta variantes anatómicas y anomalías que pueden dificultar o complicar este procedimiento. Por este motivo, el médico especialista que manipula vías venosas centrales debe estar familiarizado tanto con las técnicas de cateterización venosa como con la anatomía del sistema cardiovascular.

\section{CASO CLÍNICO}

Paciente de 76 años que ingresa en el Servicio de Cardiología por infarto agudo de miocardio de localización anterior complicado con insuficiencia cardiaca. Se decide la colocación de un catéter venoso central accediendo a través de la vena subclavia derecha.

La radiografía simple de tórax de control muestra un trayecto anómalo del catéter que

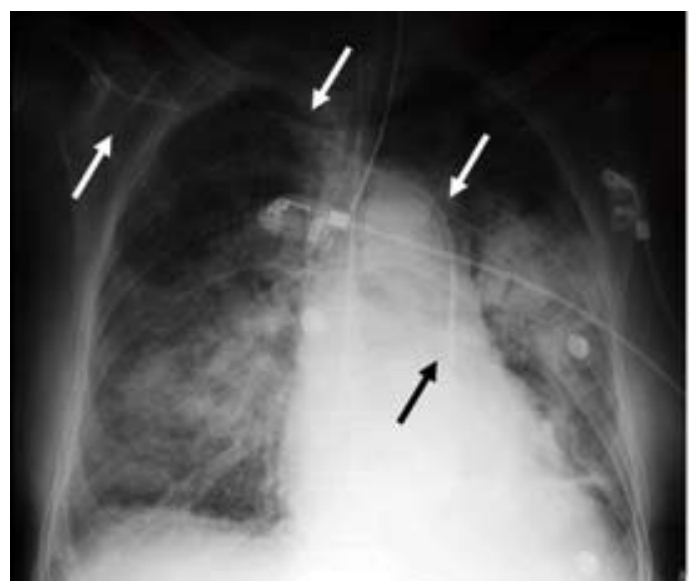

Figura 1. A. Radiografía simple de tórax. Las flechas indican el trayecto del catéter venoso. El catéter está introducido a través de vena subclavia derecha, cruza el mediastino a través de la vena braquiocefálica y desciende por el lado izquierdo hasta el seno coronario.

\section{DISCUSIÓN}

Las venas cardinales constituyen el principal sistema venoso de drenaje del desciende por el lado derecho del mediastino para desviarse posteriormente siguiendo un perfil cardiaco izquierdo (Fig. 1A).

Con el objetivo de descartar la canulación de un vaso arterial (subclavia o carótida común derechas), se solicita una gasometría ( $\mathrm{pH}: 7,35$, pO2: 40 , pCO2: 48) y se registra la curva de presión intraluminal (15 mmHg). Ambas demuestran la existencia de un flujo venoso a través de la vía.

A continuación, se implanta un catéter de Swan-Ganz en la rama principal derecha de la arteria pulmonar, también a través de la vena subclavia derecha. Se realiza un ecocardiograma que muestra dilatación del seno coronario (diámetro de 1,5 cm). En el interior del seno coronario se observa el catéter abocando a la aurícula derecha, al ventrículo derecho y, finalmente, a la arteria pulmonar. Ninguna otra vena conecta con la aurícula derecha (Fig. 1B).

Todos estos datos son compatibles con una malformación venosa que consiste en la persistencia de la VCSI con desembocadura en el seno coronario, asociada a permeabilidad de la anastomosis entre el sistema venoso derecho y el izquierdo.

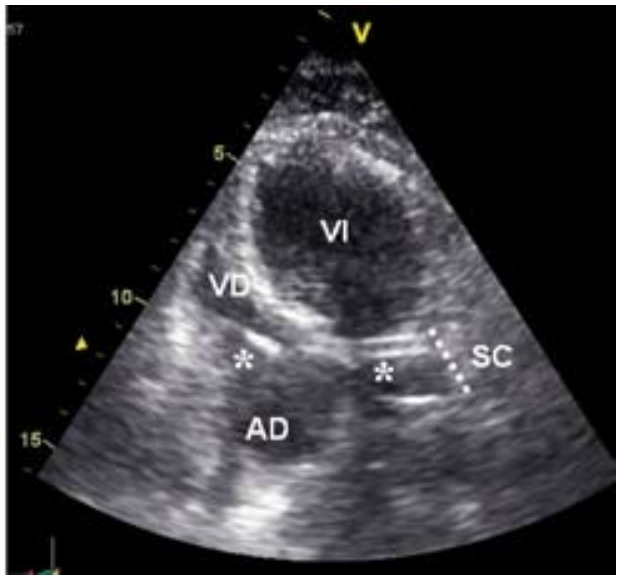

B. Ecocardiograma transtorácico. VI: ventrículo izquierdo. VD: ventrículo derecho. AD: Aurícula derecha. SC: seno coronario. Se observa el catéter de Swan-Ganz (*) discurriendo a través del $\mathrm{SC}$ que está dilatado (diámetro de $2 \mathrm{~cm}$ ). Posteriormente, el catéter atraviesa el ostium del SC desembocando en $\mathrm{AD}$ y $\mathrm{VD}$.

embrión ${ }^{1}$. Las venas cardinales anteriores drenan la parte craneal del embrión y las posteriores la parte caudal. La vena cardinal anterior y posterior de cada lado des- 
embocan en una vena cardinal primitiva que penetra en el seno venoso.

Durante la octava semana del desarrollo embrionario, las venas cardinales anteriores se conectan por una anastomosis oblicua que deriva la sangre del lado izquierdo al derecho y que se transforma en la vena braquiocefálica izquierda. Al mismo tiempo, la parte caudal de la vena cardinal anterior izquierda degenera debido a la disminución de compresión de la aurícula izquierda y del hilio pulmonar izquierdo dando lugar a la vena y el ligamento de Marshall, localizados en la cara posterior de la aurícula izquierda ${ }^{2}$. La vena cardinal anterior derecha y la vena cardinal primitiva derecha forman la vena cava superior.

Durante la formación de la vena cava superior, se producen múltiples transformaciones que predisponen a la aparición de variaciones en la edad adulta. La anomalía más frecuente, es una VCSI persistente que deriva de las venas cardinales izquierda y primitiva, con una prevalencia de hasta el $0,5 \%$ en la población general y de hasta el $10 \%$ en pacientes con cardiopatías congénitas $^{3,4}$. En el 90\% de los casos, la VCSI drena a la aurícula derecha a través del seno coronario que se encuentra dilatado ${ }^{5,6}$. En el resto, drena directamente a la aurícula izquierda dando lugar a un shunt derechaizquierda.

La persistencia de la de la VCSI puede asociarse a la obliteración de las venas cardinales derechas. Como resultado, toda la sangre del lado derecho pasa al izquierdo por la vena braquiocefálica. Sin embargo, cuando hay duplicidad de vena cava superior, la anastomosis que suele formar la vena braquiocefálica izquierda es pequeña o no existe ${ }^{7}$.

En el caso de nuestra paciente, existe una persistencia de la VCSI con permeabilidad de la anastomosis que la une con el lado derecho. Esta anomalía puede asociarse tanto a ausencia como a presencia de vena cava superior derecha (VCSD) ${ }^{8,9}$. En el caso descrito, la ausencia de VCSD determina que todo el drenaje venoso de las zonas craneales desemboque en la aurícula derecha a través del seno coronario.

La colocación de catéteres por vía venosa subclavia y yugular se asocia a complicaciones hasta en un 15\% de los casos: infecciones, lesiones vasculares, neumotórax, tromboembolia, embolia aérea, arritmias ventriculares, perforación cardiaca, taponamiento cardiaco y trayectos anómalos. Entre los trayectos anómalos del catéter central se incluyen los recorridos a través de pleura, pericardio, mediastino, canalización arterial o cateterización de una vía venosa aberrante ${ }^{2,4}$.

La sospecha diagnóstica de una vía venosa anómala surge frecuentemente tras la implantación de catéteres venosos al observar un trayecto distinto al habitual en la radiografía de tórax de control. La realización de una gasometría y la medición de la presión dentro del vaso permite distinguir entre canulación venosa o arterial. La confirmación diagnóstica y la descripción precisa de la anatomía venosa puede realizarse mediante angiografía, ecocardiografía, tomografía computerizada o cardioresonancia ${ }^{4-6}$.

Las anomalías del sistema venoso se asocian a un aumento de las complicaciones de la cateterización venosa central. Por este motivo, es esencial que el médico especialista que practica este procedimiento, conozca las variantes anatómicas más comunes para afrontar su hallazgo casual durante el manejo de los pacientes.

\section{BIBLIOGRAFÍA}

1. Paval J, KayaK S. A persistent left superior vena cava. Singapore Med J 2007; 48 (3): e90.

2. KonVICKA JJ, VILLAMARIA FJ. Images in anestesia: anesthetic implications of persistent left superior vena cava. Can J Anesth 2005; 52: 805.

3. De la Prada FJ, Sastre M, Corteza JF, Morey A, Munar MA, Alarcón A. Persistencia de la vena cava superior izquierda descubierta durante la implantación de catéter para hemodiálisis. Nefrología 2002; 2: 199-201.

4. Carrillo R, Contreras V, Salmerón P, Carvajal R, Hernández C, JuÁrez A. Vena cava superior izquierda persistente. Localización infrecuente del catéter venoso central. Cir Ciruj 2003; 71: 319-323.

5. SAmpó EA, Swieszkowski S, López GF. Vena cava superior izquierda persistente. Medicina (Buenos Aires) 2008; 68: 225.

6. Morillas P, Frutos A, Valero R, Rodríguez JA, Bertolomeu V. Persistencia de la vena cava superior izquierda. Rev Esp Cardiol 2000; 53: 1655 . 
7. Moore KL, Persaud TVN. Aparato cardiovascular. En: Embriología clínica ( $1^{\mathrm{a}}$ ed en español). Mexico: McGraw-Hill Interamericana, 1999; 2: 369-425.

8. Ratliff HL, Yousufuddin M, Lieving WR, Watson BE, Malas A, Rosencrance G et al. Persistent left superior vena cava: case reports and clinical implications. Int J Cardiol 2006; 113 : 242-246.

9. González-Juanatey C, Testa A, Vidan J, IzQuierdo R, García-Castelo A, Daniel C et al. Persistent left superior vena caba drainining into the coronary sinus: report of 10 cases and literature review. Clin Cardiol 2004; 27: 515-518. 